\title{
WILEY-VCH
}

DOI: 10.1002/ (adom.201901276)

Article type: Full Paper

\section{High responsivity and wavelength selectivity of GaN-based resonant cavity photodiodes}

Jing Li, Chao Yang, Lei Liu, Haicheng Cao, Shan Lin, Xin Xi, Xiaodong Li, Zhanhong Ma, Kaiyou Wang, Amalia Patanè and Lixia Zhao*

Jing Li, Shan Lin, Xin Xi, Xiaodong Li, Zhanhong Ma and Lixia Zhao

State Key Laboratory of Integrated Optoelectronics, Institute of Semiconductors, Chinese Academy of Sciences, No. A35, Qinghua East Road, Haidian District, Beijing, 100083, China Center of Materials Science and Optoelectronic Engineering, University of Chinese Academy of Sciences, No. 19A, Yuquan Road, Shijingshan District, Beijing 100049, China

Chao Yang, Lei Liu and Haicheng Cao

Semiconductor Lighting Research and Development Center, Institute of Semiconductors, Chinese Academy of Sciences, No. A35, Qinghua East Road, Haidian District, Beijing, 100083, China

Kaiyou Wang

State Key Laboratory of Superlattices and Microstructures, Institute of Semiconductors, Chinese Academy of Sciences, Beijing 100083, China

Amalia Patanè

School of Physics and Astronomy, University of Nottingham, Nottingham NG7 2RD, United Kingdom

*E-mail:1xzhao@semi.ac.cn

Keywords: lateral porous GaN distributed Bragg reflectors, resonant cavity, high responsivity, high wavelength selectivity, Indium Gallium Nitride

Abstract: The implementation of blue-light photodiodes based on InGaN in emerging technologies, such as free-space visible light communication (VLC), requires transformative approaches towards enhanced performance, miniaturization, and integration beyond current Sibased technologies. Here, we report on the design and realization of high-performance InGaNbased resonant cavity photodiodes with high-reflectivity lateral porous $\mathrm{GaN}$ distributed Bragg reflectors. The well-controlled porosification of $\mathrm{GaN}$ on the 2-inch wafers enables us to design and fabricate optical components, unlocking the potential of nitride semiconductors for several applications. These resonant-cavity-enhanced photodiodes, which have a $12 \mathrm{~nm}$-thick optically active region, exhibit a high responsivity $\left(\sim 0.1 \mathrm{~A} \mathrm{~W}^{-1}\right)$ to blue-light even without any externally applied voltage. Furthermore, the device can operate as both an emitter and a detector of visible 


\section{WILEY-VCH}

light at well-defined wavelengths with spectral overlap between the electroluminescence emission and photocurrent responsivity, meeting the requirement of wavelength selectivity, thermal stability and low-power consumption for VLC, with potential for integration of different functionalities, i.e. light emission and detection, on a single chip without additional light filters.

\section{Introduction}

Following the rapid development of GaN-based light emitting diodes (LEDs), visible light communication (VLC) has attracted considerable attention: ${ }^{[1]}$ VLC is secure and not restricted by electromagnetic interference or license requirements, offering a potential route to future wireless light communications beyond general lighting applications. To achieve high speed data transmission, the modulation bandwidth of the LED should be enhanced by optimization of the concentration and radiative recombination of carriers. ${ }^{[2-4]}$ For the optical receiver of a VLC system, the quantum efficiency $(Q E)$ and temporal response of the photodetector are also important as they determine data transmission distance and rate. In particular, the miniaturization and simplification of free-space VLC systems for integration of emission and detection of light on a single chip require photodetectors with high sensitivity and wavelength selectivity matching the source's emission spectrum. ${ }^{[5-8]}$ Currently, Si-based photodetectors are used to detect blue light from GaN-based white LEDs by implementing a blue-filtering technology.${ }^{[9-11]}$ However, this technology suffers from low light transmittance, high cost and complexity of the VLC system. Furthermore, the broadband response of Si-based photodetectors causes undesirable interference effects between the detected and background signals. ${ }^{[12,13]}$ Alternatively, InGaN-based photodetectors could offer an alternative solution as they have a larger optical absorption coefficient and a band gap that can be tuned by the Incomposition. ${ }^{[14-18]}$ On the other hand, their quantum efficiency tends to be limited by the deterioration of the crystal quality in structures with thick optical active regions. 


\section{WILEY-VCH}

Resonant cavities can increase the $Q E$ of InGaN photodetectors with thin absorber layers because of the increased optical field inside the cavity. ${ }^{[19,20]}$ Previous studies have reported GaN-based resonant cavity devices using different approaches (e.g. dual dielectrics, epitaxy, hybrid cavity structures, etc). ${ }^{[21-25]}$ However, due to limitations in the epitaxy and/or device fabrication, further developments are needed. On the other hand, the porosification of n-type GaN can enable the fabrication of distributed Bragg reflectors (DBRs) and their implementation in optical components. ${ }^{[26-34]}$

In this work, we report on the high-performance InGaN/GaN resonant-cavity-enhanced photodiodes (RCEPD) with high responsivity and wavelength selectivity. The resonant cavity of the photodiode comprises a top $\mathrm{Ta}_{2} \mathrm{O}_{5} / \mathrm{SiO}_{2} \mathrm{DBR}$ and a bottom lateral porous GaN DBR. By controlling the porosification of $\mathrm{n}^{+}$-doped $\mathrm{GaN}$, we realize porous $\mathrm{GaN}$ DBRs with highreflectivity (>99.5\%) and wide-stopband $(\sim 90 \mathrm{~nm})$ for optimization of the absorption, emission and integrated spectral filtering of the diode. The photodiodes can operate even without any externally applied voltages, have a narrow-band selective detection at $\lambda=466 \mathrm{~nm}$ with a responsivity of up to $0.1 \mathrm{~A} \mathrm{~W}^{-1}$, a high $Q E$ of $\sim 27.3 \%$, and a rapid and linear response, which are all desirable properties for an optical receiver. Furthermore, our resonant-cavity-enhanced photodiode can operate as both an emitter and a detector of visible light with spectral overlap between the electroluminescence emission and the photocurrent responsivity, which can offer opportunities for electro-optics and photoelectric conversion in miniaturized and energy efficient VLC systems.

\section{Experimental results and discussion}

\subsection{A resonant-cavity-enhanced InGaN photodiode with a porous GaN DBR}

Figure 1a shows the schematic layer structure and optical image (inset) of our InGaN/GaN photodiode with bottom and top DBRs, and metal contacts to the p- and n-type GaN layers. The cavity structure was designed for a main resonance at $\lambda=500 \mathrm{~nm}$ using the transfer matrix 


\section{WILEY-VCH}

method to co-optimize the blue light absorption and the light emission properties of the active region. ${ }^{[35]}$ The optical field and refractive index distribution along the growth axis $(z)$ are shown in Figure 1b. The indium tin oxide (ITO) and the InGaN/GaN multiple quantum well (MQW) regions are located at the node and antinode positions of the optical field, respectively, to reduce the absorption from the ITO layer and increase the coupling between the optical field and MQWs. A photodiode without the bottom and top mirrors was also fabricated and used as a control sample. Details of the fabrication process are in the experimental section.

Our samples were grown on the $c$-plane of a sapphire substrate by metal organic chemical vapor deposition (MOCVD), which is able to ensure accurate thickness and doping control of the epitaxial layers. The bottom mirror of the resonant cavity consists of a stack of 12 pairs of $\mathrm{n}^{+}$-doped $\mathrm{GaN}(70 \mathrm{~nm})$ and undoped $(\mathrm{u}) \mathrm{GaN}(46 \mathrm{~nm})$ layers. The stack is transformed into a porous GaN/u-GaN periodic array by electrochemical (EC) etching (Figure 1a). The refractive index of the porous $\mathrm{GaN}$ is controlled and tuned by varying the EC etching voltage. ${ }^{[36]}$ We have designed and fabricated several porous GaN DBRs and used porosification to optimize their reflectivity properties and refractive index-contrast (Supplementary Information, Figure S1 and S2). Above the $\mathrm{n}^{+}-\mathrm{GaN} / \mathrm{u}-\mathrm{GaN}$ stacked layers, a $730 \mathrm{~nm}$-thick $\mathrm{n}-\mathrm{GaN}$ layer (doped to $1 \times 10^{18}$ $\mathrm{cm}^{-3}$ ) was grown as the $\mathrm{n}$-type conductive layer followed by an InGaN/GaN (3/15nm) MQWs structure. The MQWs design was chosen for detection of blue light taking into account the Stokes' shift between the luminescence and absorption spectra. ${ }^{[37]}$ Using the InGaN QWs as absorbers instead of a single InGaN layer helps to minimize the formation of defects during the growth. Also, it increases the overlap between the InGaN absorber and the antinode of the standing wave optical field inside the cavity. Finally, a $120 \mathrm{~nm}$ p-GaN layer (doped to $3 \times 10^{18}$ $\mathrm{cm}^{-3}$ ) was grown as the p-type conductive layer. To improve current spreading, a $30 \mathrm{~nm}$-thick ITO film was deposited on the $\mathrm{p}-\mathrm{GaN}$ layer by electron beam evaporation. ${ }^{[38]}$ To minimize the absorption loss in the ITO layer and increase the photon-carrier coupling in the resonant-cavity, a phase-shift (PS) adjustment $\mathrm{SiO}_{2}$ layer was used. Its thickness (144 nm) was calculated from 


\section{WILEY-VCH}

the resonant condition $2 \beta L+\varphi_{1}+\varphi_{2}=2 m \pi$, where $m=1,2,3 \cdots, \quad \beta=2 n \pi / \lambda$ is the propagation constant, $n$ is the effective refractive index, $\varphi_{1}$ and $\varphi_{2}$ are the reflected phases for the top and bottom DBRs, respectively. ${ }^{[35]}$ The $\mathrm{SiO}_{2}$ layer was deposited on the ITO by magnetron sputtering. Subsequently, an optimized dielectric DBR consisting of 5 pairs of $\mathrm{SiO}_{2} / \mathrm{Ta}_{2} \mathrm{O}_{5}$ layers were deposited by magnetron sputtering to form the top mirror of the resonant cavity (Supplementary Information, Figure S3). Finally, a resonant cavity structure with a length of $1096 \mathrm{~nm}$ was obtained.

\subsection{High-reflectivity porous GaN DBR}

Figure 2a shows the cross-sectional scanning electron microscopy (SEM) image of the resonant cavity structure and a porous GaN DBR. The well-defined interface between porous$\mathrm{GaN}$ and undoped-GaN is achieved through the controlled modulation of the thickness and doping of the layers during the growth, as well as the high doping selectivity of the post-growth EC etching. No significant damage, such as warping and/or cracks of the layers, is observed. The porous layers comprise triangle-shaped pores, which are embedded between adjacent uniform layers of u-GaN. Furthermore, some pores take the shape of hemi circles as a result of the lateral annexation and broadening of nearby pores in $\mathrm{n}^{+}-\mathrm{GaN} .^{[30]}$ The etching occurs preferentially along specific crystal planes, as in traditional wet etching of GaN in alkali solution. ${ }^{[39]}$ Figure $2 \mathrm{~b}$ shows the reflectivity spectrum of the porous GaN DBR. The DBR has a high peak reflectivity (>99.5\%) with a wide stopband centered at $\lambda=500 \mathrm{~nm}$, which extends from $\lambda=453 \mathrm{~nm}$ to $543 \mathrm{~nm}$, covering the blue and green light emission spectrum. This is the spectral range commonly used in VLC systems. ${ }^{[40]}$

We calculate the resonant cavity modes by simulating the electric field distribution and the reflectivity of the diode using the finite-difference time-domain (FDTD) method. The simulation is based on the layered structure measured by cross-sectional SEM (Figure 2a) and the refractive index in Figure 1b. Figure 2c shows the electric field distribution $(\log |\mathrm{E}|)$ in the 


\section{WILEY-VCH}

cross-sectional plane of the diode at $\lambda=500 \mathrm{~nm}$. The electric field is mainly localized in the upper layers of the porous GaN. The intensity of the electric field in the bottom layers is almost the same as the background intensity, indicating that the porous GaN DBR acts as a good mirror at the central wavelength $\lambda=500 \mathrm{~nm}$. Furthermore, standing wave fringes can be seen between the bottom porous GaN DBR and the top $\mathrm{Ta}_{2} \mathrm{O}_{5} / \mathrm{SiO}_{2}$ dielectric DBR.

The reflectivity spectrum of the cavity in Figure 2d (black solid line) shows three resonant dips at $\lambda=465,500$ and $535 \mathrm{~nm}$, corresponding to the resonant modes of the cavity. The FWHM of the resonant mode at $\lambda=465 \mathrm{~nm}$ compares with that required in VLC systems. ${ }^{[40]}$ The three resonant wavelengths are in good agreement with the FDTD simulation (red dotted line) and the resonant modes measured by photoluminescence (PL) (blue solid line). Differences between the measured and calculated modes in the reflectivity spectra at $\lambda<450 \mathrm{~nm}$ and $\lambda>550 \mathrm{~nm}$ arise from deviations of the simulated structure from the real one, including the optical anisotropy of the porous GaN layers, which is not considered in our model. In the PL spectrum, the main resonant peak is centered at $\lambda=500 \mathrm{~nm}$. It is narrower and more intense $(\sim 12$ times $)$ than that of the control sample. Weaker peaks can be also seen at $\lambda=465$ and $535 \mathrm{~nm}$ and arise from the broad light emission of the InGaN/GaN multiple QWs (Supplementary Information, Figure S4), resulting from the large lattice and thermal mismatch between $\mathrm{InN}$ and GaN. ${ }^{[37,41-}$ 44]

\subsection{Resonant-cavity-enhanced photodetector: high responsivity and spectral selectivity}

After the electrodes deposition, we first carried out chip-on-wafer (COW) mapping tests (Supplementary Information, Figure S5) to probe the uniformity of the emission peak wavelength of the photodiodes within 0.5 -inch wafers. The distribution of the peak wavelength for the RCE photodiodes is more uniform than in the control PDs. We assign this to the wavelength selectivity of the cavity structure. Following the COW mapping test, suitable photodiodes were selected from the wafers to examine their electrical and optical properties. Figure 3a shows the current-voltage $(I-V)$ curves of both the RCEPD and control PD. The dark 


\section{WILEY-VCH}

current, $I_{d a r k}$, of the RCEPD is similar to that of the control PD in both reverse and forward bias, indicating no visible degradation of the electrical properties following the EC etching used to create the porous GaN DBR. The responsivity $\left(R=I_{p h} / P S\right)$ spectra were measured in the range $\lambda=440-550 \mathrm{~nm}$ at $V=0 \mathrm{~V}$ under optical illumination with power $P=25.8 \mathrm{~mW} \mathrm{~cm}$. Here $I_{p h}=I_{\text {light }}-I_{\text {dark }}$ is the photocurrent, $I_{\text {light }}$ is current under optical illumination, and $S$ is the in-plane area of the device. The responsivity has maxima at $\lambda=466$ and $499 \mathrm{~nm}$ (Figure 3b), in good agreement with the wavelength of the resonant cavity modes. An additional mode is also observed near $\lambda=535 \mathrm{~nm}$ (inset of Figure $3 \mathrm{~b}$ ). This is within the absorption edge of the InGaN/GaN MQWs, which tends to be weak and broad due to alloy disorder (Supplementary Information Figure S4).

The responsivity spectrum of the RCEPD has a peak $R=0.102 \mathrm{~A} \mathrm{~W}^{-1}$ at $\lambda=466 \mathrm{~nm}$ with a narrow FWHM of $13 \mathrm{~nm}$. A weaker peak $\left(R=0.013 \mathrm{~A} \mathrm{~W}^{-1}\right)$ is observed at $\lambda=499 \mathrm{~nm}$ with a FWHM of $16 \mathrm{~nm}$. The weaker responsivity at longer wavelengths is due to the decreased absorption near the band edge of the InGaN QWs. ${ }^{[37]}$ The corresponding external quantum efficiency of the RCEPD is $Q E=h c R / e \lambda$, where $h, c$ and $e$ are the Planck constant, speed of light and electron charge, respectively. The $Q E$ is shown in the inset of Figure $3 \mathrm{~b}$ : The maximum value of $Q E$ is $27.3 \%$ at $\lambda=466 \mathrm{~nm}$. This is $\sim 12$ times higher than that of the control device $(2.2 \%)$ at the same wavelength. Also, it is comparable to the $Q E$ of commercial Si-based detector-filter optical receivers and the highest reported value of $Q E$ for InGaN blue detectors with a absorber thickness of about $0.5 \mu \mathrm{m}, 40$ times thicker than the $\mathrm{InGaN}$ active region of our RCEPD. ${ }^{[40,45,46]}$ Thus the RCEPD can meet the requirement of high sensitivity and narrowband detection of blue light for VLC, overcoming the limitations (e.g. background light and noise) of conventional structures, which employ a blue-filtering technology.

The $I-V$ curves of the RCEPD were measured at different light powers $P$. For these experiments, a laser beam was focused by a convex lens on the device to a spot diameter of 


\section{WILEY-VCH}

about $100 \mu \mathrm{m}$. Figure $3 \mathrm{c}$ shows the $P$-dependent $I-V$ characteristics of the RCEPD at $\lambda=466$ $\mathrm{nm}$. The dark current increases with increasing negative bias, but remains small, demonstrating good rectification properties. Under illumination, the current increases with increasing $P$ and the $I-V$ curves remain relatively flat in reverse bias. Also, the RCEPD maintains a high photoresponsivity even without any externally applied voltage, indicating an effective extraction of the photogenerated carriers by the built-in electric field of the junction.

The photocurrent $I_{p h}$ depends linearly on $P$ (Supplementary Information, Figure S6), leading to a responsivity $R$ and specific detectivity $D^{*}=R S^{1 / 2} /\left(2 e I_{\text {dark }}\right)^{1 / 2}$ that are only weakly dependent on $P$ (Figure 3d-e). ${ }^{[47]}$ As shown in Figure 3d, $R$ increases monotonically with increasing reverse bias. This behavior is due to the corresponding increase of the electric field in the intrinsic region, shortening the carrier transit and recombination times (Supplementary Information, Figure S7). As shown in Figure 3e, the specific detectivity $D^{*}$ reaches a maximum value of $8.4 \times 10^{11}$ Jones at $V=-2 \mathrm{~V}$. For $V<-2 \mathrm{~V}, D^{*}$ decreases due to the increase of the dark current. Therefore, the large RCEPD response depends linearly on the light intensity, which satisfies the requirement for linear detection of visible light and photoelectric signal conversion without signal distortion.

To evaluate the response time of the RCEPD, the device was illuminated with a LED $(\lambda=$ $470 \mathrm{~nm}, \mathrm{FWHM} \sim 20 \mathrm{~nm}$ ) powered by a square-wave signal generator. The dynamic responses were recorded using a current amplifier and an oscilloscope for different modulation frequencies and applied biases $V$ (Supplementary Information, Figure S8 and S9). As shown in Figure 3f, the dynamic response of the current is well described by $I(t)=I_{0}\left[1-\exp \left(-t / \tau_{r}\right)\right]$ and $I$ $(t)=I_{0} \exp \left(-t / \tau_{d}\right)$, where $\tau_{r}$ and $\tau_{d}$ are the time constants for the rise and decay of the current. By fitting the rising and falling edges, we derive $\tau_{r}=35 \mu$ s and $\tau_{d}=30 \mu \mathrm{s}$ at $V=0 \mathrm{~V}$. The measured response time depends on the applied reverse bias and is primarily limited by the $R C$-time of the RCPED and the transit time of the carriers across the depletion region of the junction (Supplementary Information, Figure S9). We envisage future developments by optimizing the 


\section{WILEY-VCH}

layout of the device as well as reducing parasitic capacitance and resistance for fast, large data transmission in VLC. In particular, the high saturation electron velocity of nitrides $\left(>2 \times 10^{7} \mathrm{~cm}\right.$ $\mathrm{s}^{-1}$ ) offers opportunities for fast transmission beyond that enabled by traditional $\mathrm{Si}$ photodiodes. $^{[48]}$

\subsection{Resonant-cavity-enhanced emitter: wavelength selectivity and thermal stability}

The light emitting properties of the photodiode are significantly improved by the implementation of the DBRs. Figure $4 \mathrm{a}$ and $\mathrm{b}$ show the electroluminescence (EL) spectra of the control and RCE devices for different injected currents. For $I=1 \mathrm{~mA}$, the EL emission of the control device is peaked at $\lambda=512 \mathrm{~nm}$, corresponding to the PL peak wavelength of the epitaxial structure (Supplementary Information, Figure S4). With increasing current from 1 to $40 \mathrm{~mA}$, the EL emission shifts from $512 \mathrm{~nm}$ to $487 \mathrm{~nm}$ due to the screening of the quantum-confined Stark effect by the injected carriers. ${ }^{[3,41,49]}$ A further increase of current up to $100 \mathrm{~mA}$ induces a small red-shift of the EL band to $494 \mathrm{~nm}$ due to self-heating. ${ }^{[50]}$ In contrast, the EL spectrum consists of three peaks centered at $\lambda=466,497$ and $529 \mathrm{~nm}$, which do not blue-shift with increasing current. These peaks correspond to the designed resonant cavity modes (Figure $2 \mathrm{~d}$ ). In addition, only a small red-shift $(\sim 1 \mathrm{~nm})$ of the main resonant peak is observed with increasing current from $I=1$ to $100 \mathrm{~mA}$ (Figure 4c), indicating an improved wavelength stability of the RCEPD, an essential requirement for reliable data communication. The FWHM of the main EL peak is $\sim 7 \mathrm{~nm}$, which is much narrower than that of the control sample (FWHM $\sim 42 \mathrm{~nm}$, Figure 4d). The three EL emission modes also coincide with the PL peaks with a small difference between their positions due to different excitation conditions.

\section{Conclusion}

In conclusion, we have demonstrated high-performance InGaN/GaN resonant-cavityenhanced photodiodes that make use of $\mathrm{Ta}_{2} \mathrm{O}_{5} / \mathrm{SiO}_{2}$ and lateral porous $\mathrm{GaN}$ DBRs. The bottom porous GaN DBR has a high reflectivity (>99.5\%), a wide stopband (band width of $90 \mathrm{~nm}$ ) and 


\section{WILEY-VCH}

well-defined resonant cavity modes. The photodiodes can operate without any externally applied voltage, have a narrow-band selective detection at $\lambda=466 \mathrm{~nm}$ with a responsivity of up to $0.1 \mathrm{~A} \mathrm{~W}^{-1}$, and a rapid and linear response, all desirable properties for an optical receiver. Furthermore, we have shown that the resonant cavity improves the light emitting properties of the photodiode, which can operate as a light emitting source at well-defined wavelengths under different current injection conditions. Thus, the GaN-based resonant-cavity-enhanced optoelectronic devices presented in this work offer opportunities to realize high-performance detectors and emitters with potential for large data communications bandwidth that can deliver increased functionality and low power consumption. With further improvement of InGaN crystal qualtiy, these GaN-based resonant-cavity-enhanced photodiodes can offer a route to new technologies for visible light communication beyond Si-based technologies. In particular, the tunability of the refractive index of porous $\mathrm{GaN}$ via a well-controlled porosification method offers opportunities to design and fabricate novel optical components and unlock the potential of nitride semiconductors for several applications, beyond the capability of traditional optoelectronic devices in the current literature.

\section{Experimental Section}

Epitaxial growth. The InGaN/GaN multi-layer structure was grown on a 2 inch $c$-face sapphire substrate by MOCVD. The sample contains 12 pairs of $\mathrm{n}^{+}-\mathrm{GaN} / \mathrm{u}-\mathrm{GaN}$ periodic stacks embedded within an InGaN/GaN epitaxial multi-layer structure. The $\mathrm{n}^{+}-\mathrm{GaN} / \mathrm{u}-\mathrm{GaN}$ periodic stack was grown on a $500 \mathrm{~nm}$ thick u-GaN layer and was transformed into a porous $\mathrm{GaN} / \mathrm{u}-$ GaN DBR by electrochemical (EC) etching (Supplementary Information S1). Under the 500 $\mathrm{nm}$ thick $\mathrm{u}-\mathrm{GaN}$ layer, a $30 \mathrm{~nm}$ nucleation layer was grown, followed by a $1 \mu \mathrm{m}$ thick $\mathrm{u}-\mathrm{GaN}$ buffer layer. Also, a $600 \mathrm{~nm}$ thick $\mathrm{n}^{+}-\mathrm{GaN}$ layer $\left(5 \times 10^{18} \mathrm{~cm}^{-3}\right)$ was grown as a current spreading layer to ensure a uniform distribution of the anodization voltage across the sample during the EC etching. The epitaxial structure above the porous GaN/u-GaN DBR consists of a $730 \mathrm{~nm} n-$ 


\section{WILEY-VCH}

GaN layer (doped to $1 \times 10^{18} \mathrm{~cm}^{-3}$ ), 4 pairs of InGaN/GaN (3/15 nm) multiple-quantum wells (MQWs), and a $120 \mathrm{~nm} \mathrm{p}-\mathrm{GaN}$ layer (doped to $3 \times 10^{18} \mathrm{~cm}^{-3}$ ).

Fabrication of the porous GaN DBR by electrochemical (EC) etching. Following the epitaxial growth and before the EC etching of the $\mathrm{n}^{+}-\mathrm{GaN} / \mathrm{u}-\mathrm{GaN}$ periodic stack, a $200 \mathrm{~nm}$ thick $\mathrm{SiO}_{2}$ layer was deposited to prevent the etching of the wafer surface. Hence, 300- $\mu$ m-wide stripes were patterned on the wafer by photolithography. To expose the stack structure sidewalls, the openings between the stripes were etched down to the $500 \mathrm{~nm}$ thick u-GaN layer by inductively coupled plasma (ICP) etching. To convert the $\mathrm{n}^{+}-\mathrm{GaN}$ layer into porous $\mathrm{GaN}$, the $\mathrm{EC}$ etching was carried out in a $\mathrm{NaOH}(1 \mathrm{M})$ solution at a voltage of $8 \mathrm{~V}$ using a high precise Keithley 2400 power supply, which is helpful to the stability of the etching voltage. In addition, a magnetic stirrer was used in the EC etching to promote the diffusion of ions into the porous GaN channels and the discharge of bubbles generated in the reaction.

Fabrication of the photodiodes. A mesa area with diameter $d=200 \mu \mathrm{m}$ was patterned by standard photolithography and ICP etching, followed by the opening of an aperture with diameter $d=100 \mu \mathrm{m}$ on the $\mathrm{SiO}_{2}$ layer using a BOE solution. To improve the current spreading property, a $30 \mathrm{~nm}$ ITO film was deposited by electron beam evaporation on the exposed p-GaN and annealed at $550{ }^{\circ} \mathrm{C}$ under nitrogen atmosphere for 30 minutes to improve the ohmic contact. Then, $\mathrm{Cr} / \mathrm{Al} / \mathrm{Ti} / \mathrm{Au}$ layers were deposited by electron beam evaporation to form the p-type and n-type electrodes. The electrodes were defined by standard lithography and lift-off techniques. To minimize the light absorption from the ITO layer and increase the photon-carrier coupling, a phase-shift adjustment $\mathrm{SiO}_{2}$ layer was also deposited on the ITO by magnetron sputtering. Finally, 5 pairs of $\mathrm{Ta}_{2} \mathrm{O}_{5} / \mathrm{SiO}_{2}$ dielectric stacks were deposited by magnetron sputtering with a quarter-wavelength pair of $\mathrm{Ta}_{2} \mathrm{O}_{5}(57.6 \mathrm{~nm})$ and $\mathrm{SiO}_{2}(85.6 \mathrm{~nm})$ to act as both the top Bragg reflector and the detection window covering from 440 to $550 \mathrm{~nm}$ of the incident light.

Measurement setup. The reflectivity spectra of the porous GaN DBR, the EC etched wafer, the $\mathrm{Ta}_{2} \mathrm{O}_{5} / \mathrm{SiO}_{2}$ dielectric $\mathrm{DBR}$, and the optical resonant cavity were measured using a 


\section{WILEY-VCH}

UV-vis-near-infrared spectrophotometer (Varian Cary-5000). The photoluminescence (PL) measurements were carried at room temperature in a reflection geometry with excitation and detection of light along the growth axis. The excitation source was provided by a Diode Pumped Solid State laser of wavelength $\lambda=355 \mathrm{~nm}$. The laser was focussed onto the sample to a spot diameter $d \sim 2 \mu \mathrm{m}$. The EL spectra were measured at room temperature using an integrating sphere in the continuous-wave current mode. The photoresponse of the photodetector was measured using an Agilent semiconductor parameter analyzer B1500A, and the light output from a supercontinuum laser source (Fianium, WhiteLase CS390-1.5 Blue-UV Supercontinuum) was directed into a monochromator for single wavelength selection. The continuous output wavelength of this supercontinuum laser source ranges from 390 to $2400 \mathrm{~nm}$. The incident optical power was measured using a calibrated power meter (Thorlabs GmbH., PM 100D). The power dependent response was measured using an attenuator to change the incident light power. The time-resolved high frequency response characteristics of the RCEPD were investigated by a digital oscilloscope, a power amplifier and a pulsed light source. Pulsed light was generated by a blue LED driven by a square-wave signal generator.

\section{Supporting Information}

Supporting Information is available from the Wiley Online Library or from the author.

\section{Acknowledgements}

This work was supported by the National Natural Science Foundation of China under Grants 61774148 and 11574306, and the National Basic Research and High Technology Program of China under Grants 2017YFB0403601 and 2017YFB0403602. AP acknowledges the Chinese Academy of Sciences (CAS) for the Award of a "President's International Fellowship for Visiting Scientists".

Received: ((will be filled in by the editorial staff))

Revised: ((will be filled in by the editorial staff)) Published online: ((will be filled in by the editorial staff))

\section{References}

[1] J. Y. Tsao, M. H. Crawford, M. E. Coltrin, A. J. Fischer, D. D. Koleske, G. S. Subramania, G. T. Wang, J. J. Wierer, R. F. Karlicek Jr, Adv. Opt. Mater. 2014, 2, 809. 


\section{WILEY-VCH}

[2] S. C. Zhu, Z. G. Yu, L. X. Zhao, J. X. Wang, J. M. Li, Opt. Express 2015, 23, 13752.

[3] S. Zhu, S. Lin, J. Li, Z. Yu, H. Cao, C. Yang, J. Li, L. Zhao, Appl. Phys. Lett. 2017, 111, 171105.

[4] S. Lin, H. Cao, J. Li, X. Sun, H. Xiu, L. Zhao, Appl. Phys. Express, 2018, 11, 122101.

[5] P. H. Pathak, X. Feng, P. Hu, P. Mohapatra, IEEE Commun. Surv. Tut. 2015, 17, 2047.

[6] C. Sun, M. T. Wade, Y. Lee, J. S. Orcutt, L. Alloatti, M. S. Georgas, A. S. Waterman, J. M. Shainline, R. R. Avizienis, S. Lin, B. R. Moss, R. Kumar, F. Pavanello, A. H. Atabaki, H. M. Cook, A. J. Ou, J. C. Leu, Y. H. Chen, K. Asanovic, R. J. Ram, M. A. Popovic, V. M. Stojanovic, Nature 2015, 528, 534.

[7] H. Shin, J. A. Cox, R. Jarecki, A. Starbuck, Z. Wang, P. T. Rakich, Nat. Commun. 2015, $6,6427$.

[8] Y. Wang, X. Wang, B. Zhu, Z. Shi, J. Yuan, X. Gao, Y. Liu, X. Sun, D. Li, H. Amano, Light Sci. Appl. 2018, 7, 83.

[9] C. W. Chow, C. H. Yeh, Y. F. Liu, Y. Liu, Electron. Lett. 2011, 47, 867.

[10] C. H. Yeh, Y. L. Liu, C. W. Chow, Opt. Express 2013, 21, 26192.

[11] R. Zhang, H. Lin, Y. Yu, D. Chen, J. Xu, Y. Wang, Laser Photonics Rev., 2014, 8, 158.

[12] J. Vucic, C. Kottke, S. Nerreter, K. D. Langer, J. W.Walewski, J. Lightwave Technol. 2010, $28,3512$.

[13] A. M. K. G. Cossu, P. Choudhury, R. Corsini, E. Ciaramella, Opt. Express 2012, 20, B501.

[14] E. Miyazaki, S. Itami, T. Araki, Rev. Sci. Instrum. 1998, 69, 3751.

[15] J. Pereiro, C. Rivera, Á. Navarro, E. Muñoz, R. Czernecki, S. Grzanka, M. Leszczynski, IEEE J. Quantum Elec. 2009, 45, 617.

[16] E. A. Berkman, N. A. El-Masry, A. Emara, S. M. Bedair, Appl. Phys. Lett. 2008, 92, 101118. 


\section{WILEY-VCH}

[17] Z. Y. Al Balushi, K. Wang, R. K. Ghosh, R. A. Vilá, S. M. Eichfeld, J. D. Caldwell, X. Qin, Y. C. Lin, P. A. DeSario, G. Stone, S. Subramanian, D. F. Paul, R. M. Wallace, S. Datta, J. M. Redwing, J. A. Robinson, Nat. Mater. 2016, 15, 1166.

[18] C. Rivera, J. L. Pau, Á. Navarro, E. Muñoz, IEEE J. Quantum Elec. 2006, 42, 51.

[19] H. Song, L. Guo, Z. Liu, K. Liu, X. Zeng, D. Ji, N. Zhang, H. Hu, S. Jiang, Q. Gan, Adv. Mater. 2014, 26, 2737.

[20] Y. Wang, X. Li, V. Nalla, H. Zeng, H. Sun, Adv. Funct. Mater. 2017, 27, 1605088.

[21] C. Zhang, R. Elafandy, J. Han, Appl. Sci. 2019, 9 (8), 1593.

[22] Y. K. Song, M. Diagne, H. Zhou, A. V. Nurmikkoa, R. P. Schneider, T. Takeuchi, Appl. Phys. Lett. 2000, 77, 1744.

[23] J. M. Redwing, D. A. S. Loeber, N. G. Anderson, M. A. Tischler, J. S. Flynn, Appl. Phys. Lett. 1996, $69(1), 1$.

[24] T. C. Lu, C. C. Kao, H. C. Kuo, G. S. Huang, S. C. Wanga, Appl. Phys. Lett. 2008, 92, 141102.

[25] J. F. Carlin, J. Dorsaz, E. Feltin, R. Butté, N. Grandjean, M. Ilegems, M. Laügt, Appl. Phys. Lett. 2005, 86, 031107.

[26] C. Yang, L. Liu, S. Zhu, Z. Yu, X. Xi, S. Wu, H. Cao, J. Li, L. Zhao, J. Phys. Chem. C 2017, 121,7331 .

[27] L. Liu, C. Yang, A. Patane, Z. Yu, F. Yan, K. Wang, H. Lu, J. Li, L. Zhao, Nanoscale 2017, 9,8142 .

[28] C. Yang, X. Xi, Z. Yu, H. Cao, J. Li, S. Lin, Z. Ma, L. Zhao, ACS Appl. Mater. Interfaces 2018, 10,5492 .

[29] S. M. Lee, S. H. Gong, J. H. Kang, M. Ebaid, S. W. Ryu, Y. H. Cho, Opt. Express 2015, $23,11023$.

[30] G. Yuan, K. Xiong, C. Zhang, Y. Li, J. Han, ACS Photonics 2016, 3, 1604.

[31] S. Chen, C. Zhang, J. Lee, J. Han, A. Nurmikko, Adv. Mater. 2017, 29, 1604781. 


\section{WILEY-VCH}

[32] B. C. Shieh, Y. C. Jhang, K. P. Huang, W. C. Huang, J. J. Dai, C. F. Lai, C. F. Lin, Appl. Phys. Express 2015, 8, 082101.

[33] G. Y. Shiu, K. T. Chen, F. H. Fan, K. P. Huang, W. J. Hsu, J. J. Dai, C. F. Lai, C. F. Lin, Sci. Rep. 2016, 6, 29138.

[34] S. M. Mishkat-Ul-Masabih, A. A. Aragon, M. Monavarian, T. S. Luk, D. F. Feezell, Appl. Phys. Express 2019, 12, 036504.

[35] J. A. Jervase, H. Bourdoucen, IEEE J. Quantum Elec. 2000, 36, 325.

[36] C. Zhang, S. H. Park, D. Chen, D. W. Lin, W. Xiong, H. C. Kuo, C. F. Lin, H. Cao, J. Han, ACS Photonics 2015, 2, 980.

[37] R. W. Martin, P. G. Middleton, K. P. O’Donnell, W. Van der Stricht, Appl. Phys. Lett. 1999, 74, 263.

[38] T. C. Lu, S. W. Chen, T. T. Wu, P. M. Tu, C. K. Chen, C. H. Chen, Z. Y. Li, H. C. Kuo, and S. C. Wang, Appl. Phys. Lett. 2010, 97, 071114.

[39] K. Al-Heuseen, M. R. Hashim, and N. K. Ali, Appl. Surf. Sci. 2011, 257, 6197.

[40] S. W. Wang, F. Chen, L. Liang, S. He, Y. Wang, X. Chen, W. Lu, IEEE Wirel. Commun. 2015, 22, 61 .

[41] S. Chichibu, T. Azuhata, T. Sota, S. Nakamura, Appl. Phys. Lett. 1996, 69, 4188.

[42] J. K. Son, T. Sakong, S. N. Lee, H. S. Paek, H. Ryu, K. H. Ha, O. Nam, Y. Park, J. S. Hwang, Y. H. Cho, Appl. Phys. Lett. 2007, 90, 051918.

[43] K. S. Kim, J. K. Son, S. N. Lee, Y. J. Sung, H. S. Paek, H. K. Kim, M. Y. Kim, K. H. Ha, H. Y. Ryu, O. H. Nam, T. Jang, Y. J. Park, Appl. Phys. Lett. 2008, 92, 101103.

[44] A. Satake, Y. Masumoto, T. Miyajima, T. Asatsuma, F. Nakamura, Masao Ikeda, Phys. Rev. B 1998, 57, R2041.

[45] Hamamatsu, Inc., Si pin photodiodes for visible to near IR, precision photometry, https://www.hamamatsu.com/resources/pdf/ssd/s1223_series_kpin1050e.pdf. 


\section{WILEY-VCH}

[46] C. L. Yu, C. H. Chen, S. J. Chang, Y. K. Su, S. C. Chen, P. C. Chang, P. C. Chen, M. H. Wu, H. C. Chen, K. C. Su, IEEE Photonic. Tech. L. 2005, 17, 875.

[47] F. Yan, L. Zhao, A. Patanè, P. Hu, X. Wei, W. Luo, D. Zhang, Q. Lv, Q. Feng, C. Shen, K. Chang, L. Eaves, K. Wang, Nanotechnology 2017, 28, 27 LT01.

[48] B. E. Foutz, S. K. O’Leary, M. S. Shur, L. F. Eastman, J. Appl. Phys. 1999, 85, 7727.

[49] P. Waltereit, O. Brandt, A. Trampert, H. T. Grahn, J. Menniger, M. Ramsteiner, M. Reiche, K. Ploog, Nature 2000, 406, 865.

[50] D. Li, R. Cheng, H. Zhou, C. Wang, A. Yin, Y. Chen, N. O. Weiss, Y. Huang, X. Duan, Nat. Commun. 2015, 6, 7509. 


\section{WILEY-VCH}

a

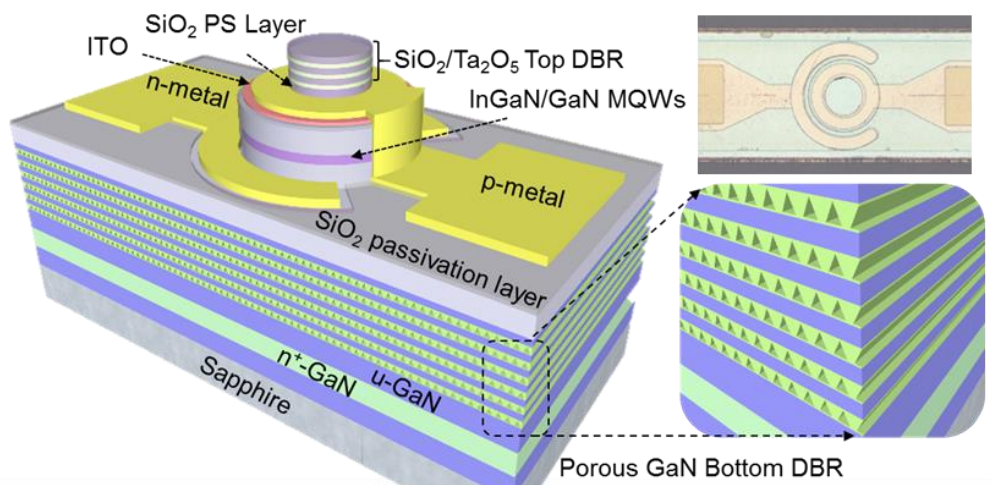

b

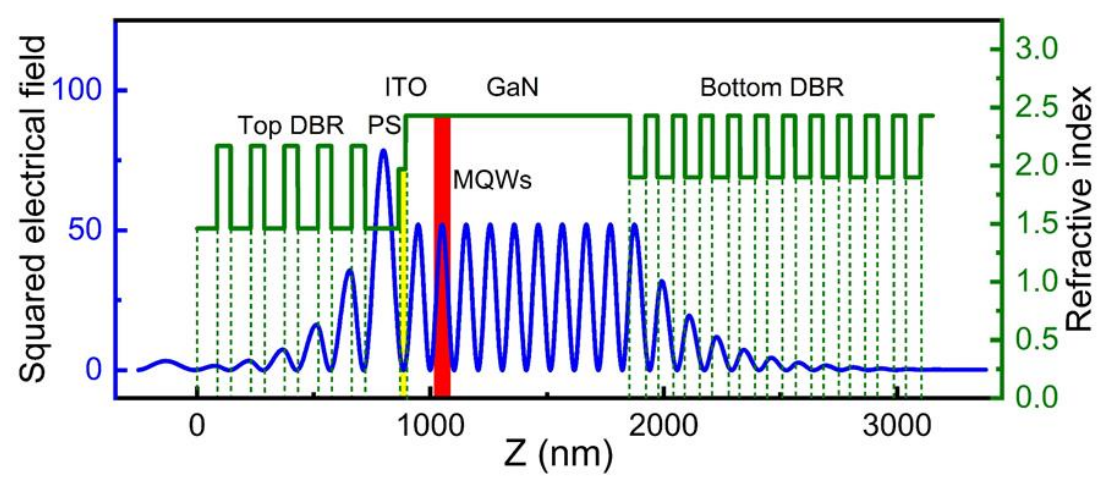

Figure 1. An InGaN-based resonant-cavity-enhanced photodiode with a lateral porous $\mathrm{GaN}$ DBR. (a) Schematic of a photodiode containing a lateral porous GaN DBR. The inset is the optical image of the diode with bottom and top DBRs, and metal contacts to the p- and n-type GaN layers. (b) Optical field and refractive index along the growth axis $(z)$ of the diode. The red and yellow areas indicate the position of the $\mathrm{InGaN} / \mathrm{GaN}$ multiple quantum wells (MQWs) and ITO, respectively. 


\section{WILEY-VCH}

a

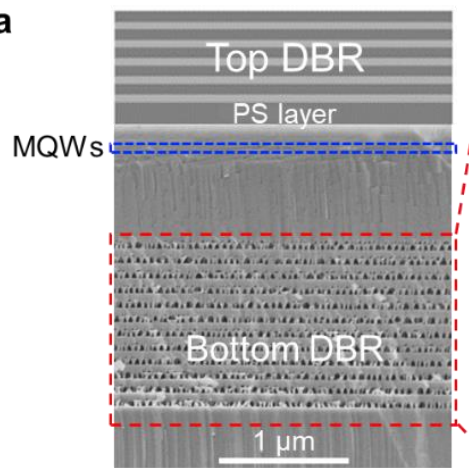

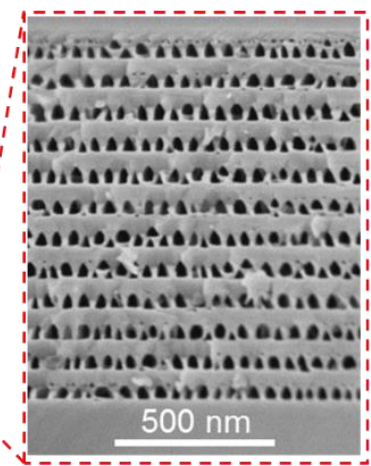

c

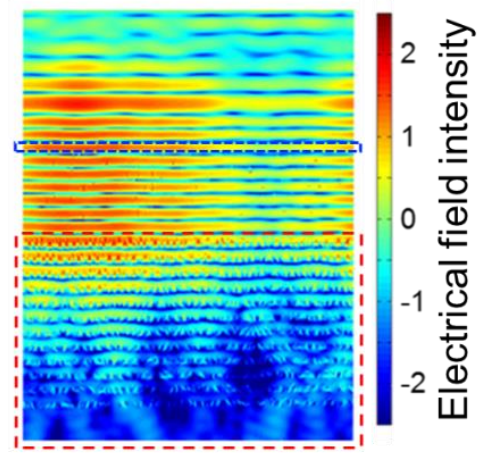

b

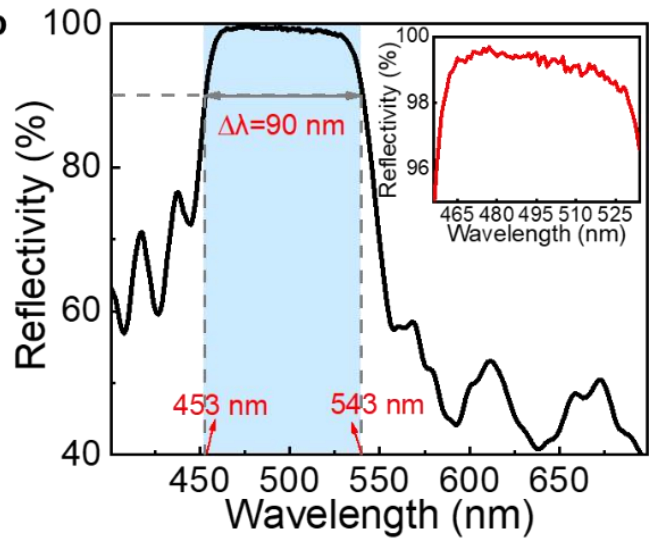

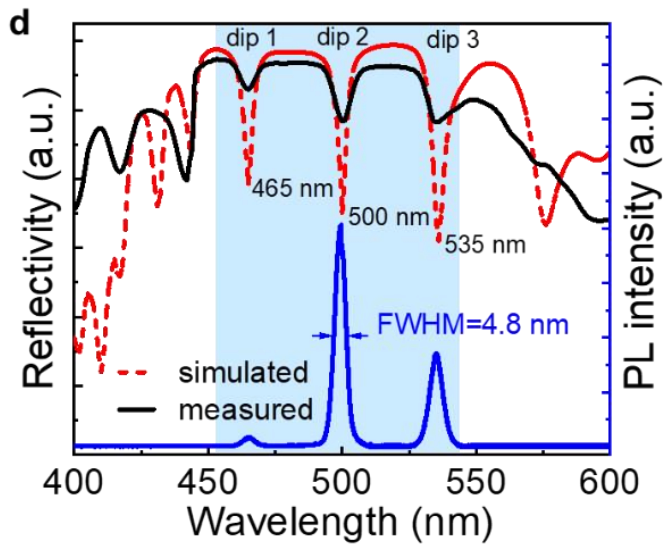

Figure 2. Resonant-cavity-enhanced photodiode: morphology and optical properties. (a) Crosssectional SEM images of the InGaN photodiode. Inset: bottom porous GaN DBR. (b) Reflectivity spectrum of the porous GaN DBR. The inset is the close-up of the spectrum with a peak reflectivity $>99.5 \%$. (c) Simulated electric field distribution $(\log |E|)$ in the cross-sectional plane of the diode at $\lambda=500 \mathrm{~nm}$. The red and blue dashed lines in (a) and (c) indicate the position of the porous GaN DBR and multiple quantum wells (MQWs), respectively. (d) Reflectivity (black: data, red: simulation) and photoluminescence (PL) (blue) spectra of the diode. Shaded light blue regions in (b) and (d) indicate the stopband of the cavity. 


\section{WILEY-VCH}
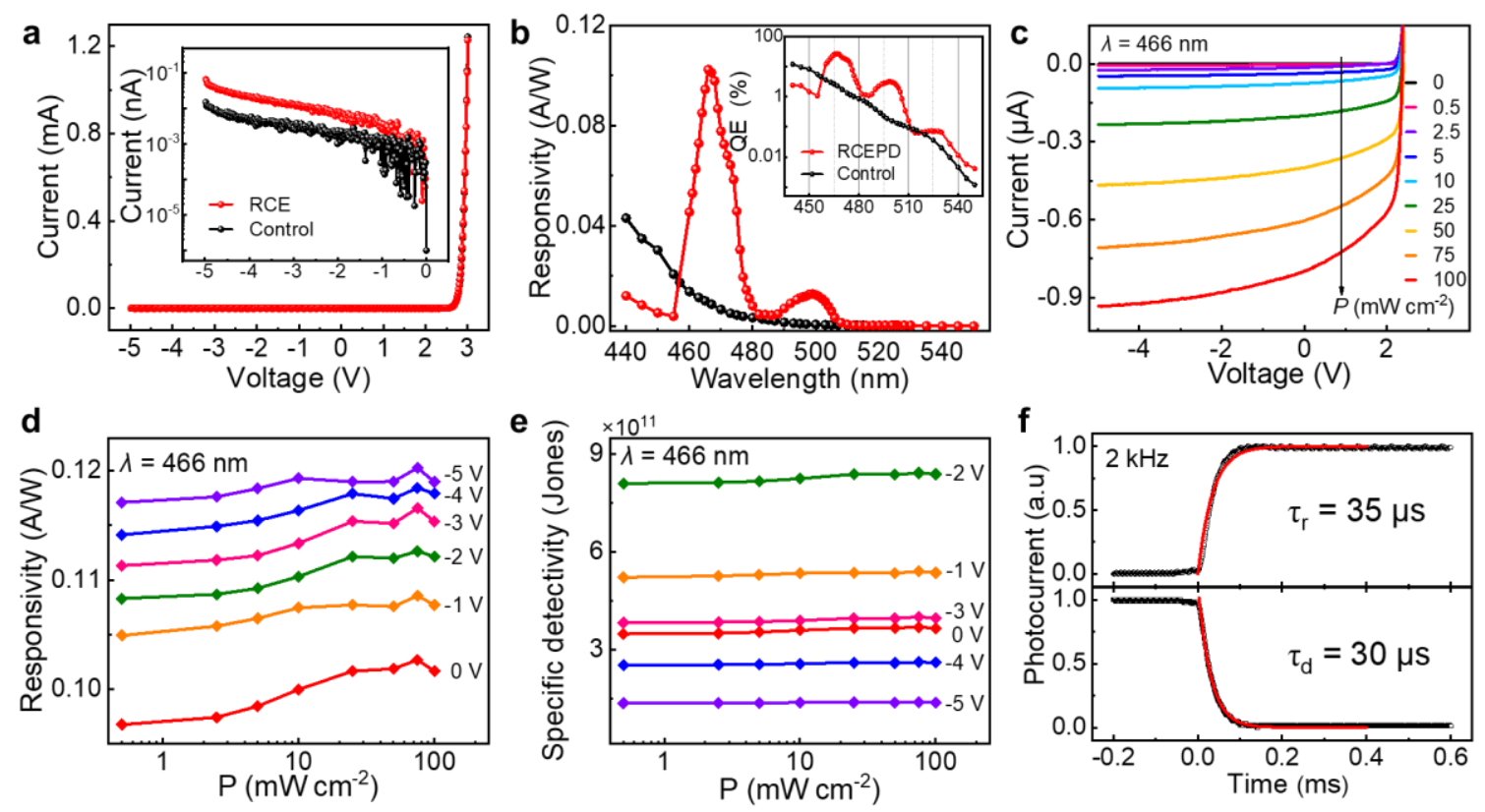

Figure 3. Photoresponse of the resonant-cavity-enhanced InGaN photodiode (RCEPD). (a) Current-voltage $I$ - $V$ curves in the dark of the RCEPD (red) and control PD (black). Inset: $\log (I)$ - $V$ curves in reverse bias. (b) Responsivity and quantum efficiency, $Q E$ (inset) versus photon wavelength at $V=0 \mathrm{~V}$ for the RCEPD and control PD. (c) $I-V$ curves of the RCEPD at $\lambda=466$ nm for powers $P$ from 0 to $100 \mathrm{~mW} \mathrm{~cm}^{-2}$. Responsivity (d) and specific detectivity (e) versus $P$ at $\lambda=466 \mathrm{~nm}$ and $V=0,-1,-2,-3,-4,-5 \mathrm{~V}$. (f) Temporal response of the photocurrent of the RCEPD under optical illumination with a $2 \mathrm{kHz}$ light-switching frequency. Red lines are fits to the data. 


\section{WILEY-VCH}
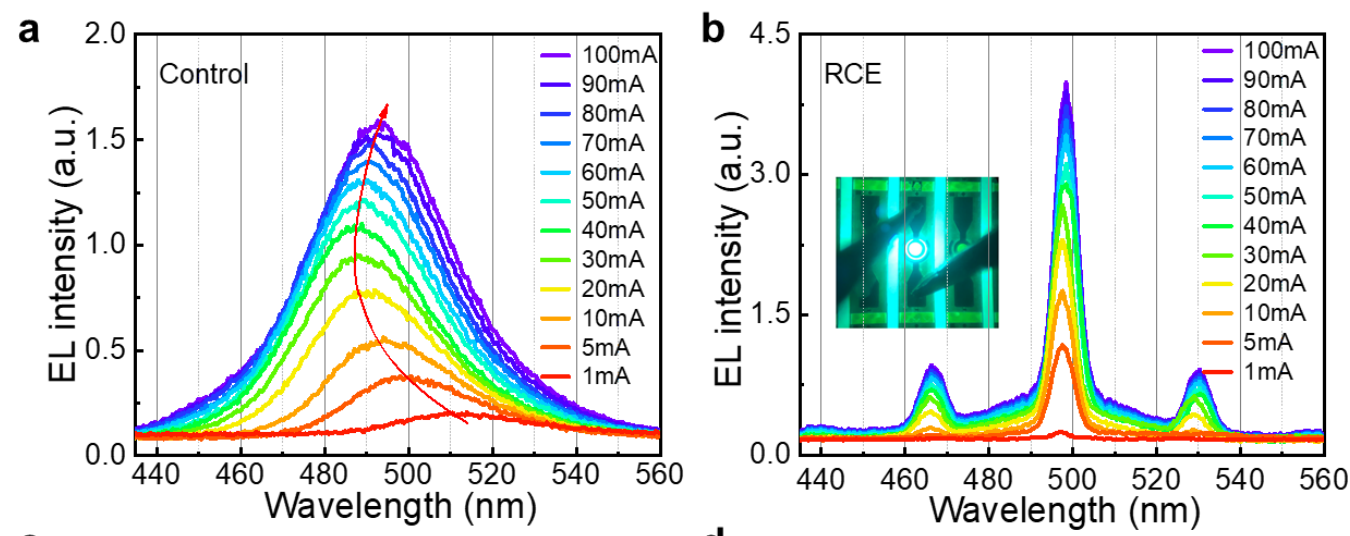

C
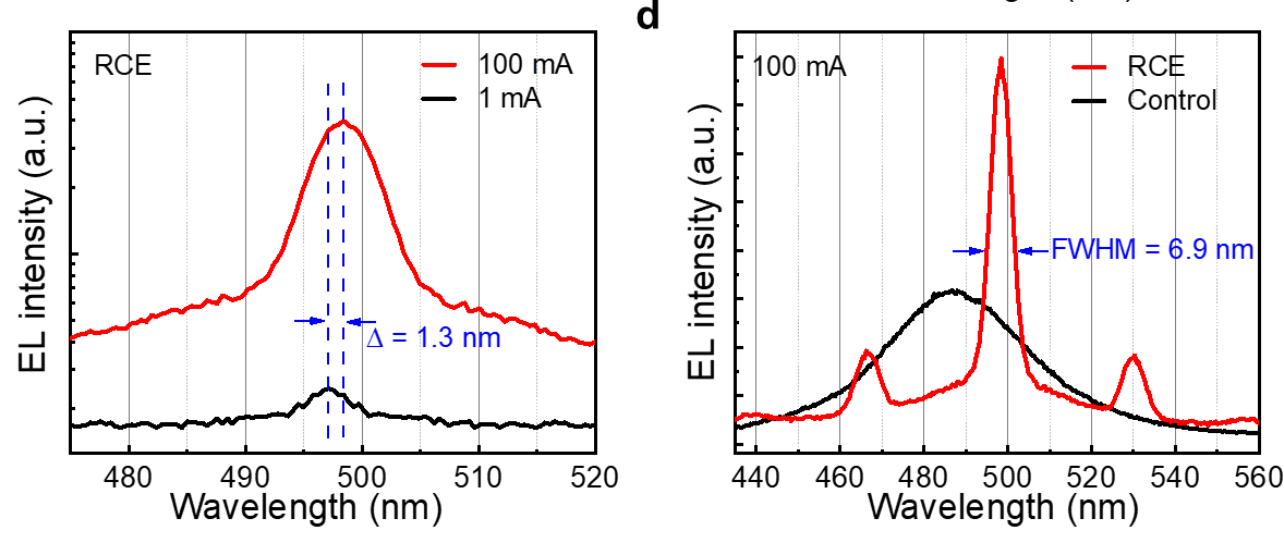

Figure 4. Light emitting characteristics of the resonant-cavity-enhanced InGaN photodiode. EL emission spectra of the (a) control and (b) RCE diodes for different currents. The red arrow is a guide to the EL peak in the control diode. The inset in part (b) shows an optical image of the RCE diode. (c) Red-shift of the EL emission peak for the RCE device with increasing current from $I=1$ to $100 \mathrm{~mA}$. (d) Comparison of the EL emission spectra of the control and RCE devices for an injection current $I=100 \mathrm{~mA}$. 


\section{WILEY-VCH}

The well-controlled porosification of $\mathrm{GaN}$ enables us to realize porous GaN distributed Bragg reflectors with high reflectivity for optimization of the absorption, emission and integrated spectral filtering of an InGaN photodiode. The dual functionality of this resonantcavity-enhanced photodiode for detection and emission of visible light, and its high performance offer opportunities in miniaturized and energy efficient visible light communication systems.

Keyword: lateral porous GaN distributed Bragg reflectors, resonant cavity, high responsivity, high wavelength selectivity, Indium Gallium Nitride

Jing Li, Chao Yang, Lei Liu, Haicheng Cao, Shan Lin, Xin Xi, Xiaodong Li, Zhanhong Ma, Kaiyou Wang, Amalia Patanè and Lixia Zhao*

High responsivity and wavelength selectivity of GaN-based resonant cavity photodiodes
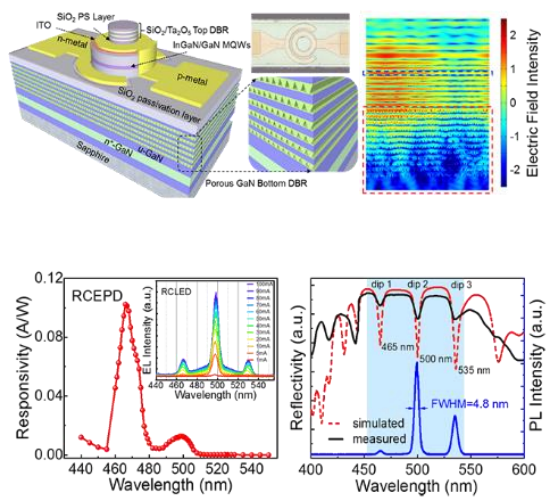


\section{WILEY-VCH}

\section{Supporting Information}

\section{High responsivity and wavelength selectivity of GaN-based resonant cavity photodiodes}

Jing Li, Chao Yang, Lei Liu, Haicheng Cao, Shan Lin, Xin Xi, Xiaodong Li, Zhanhong Ma, Kaiyou Wang, Amalia Patanè and Lixia Zhao*

\section{S1. Design and fabrication of the bottom porous GaN DBR}

a

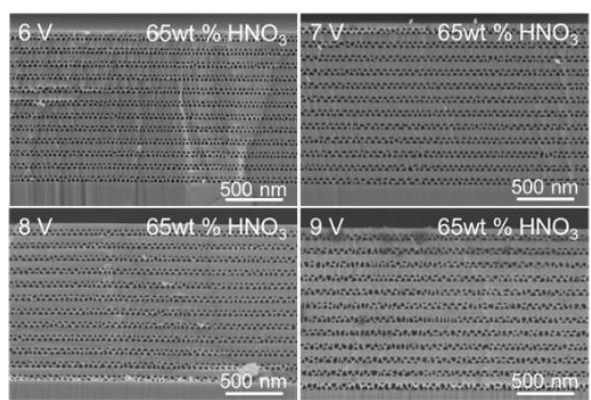

C

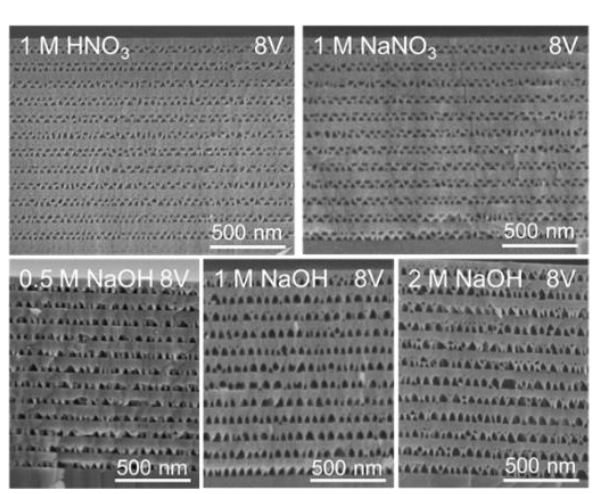

b
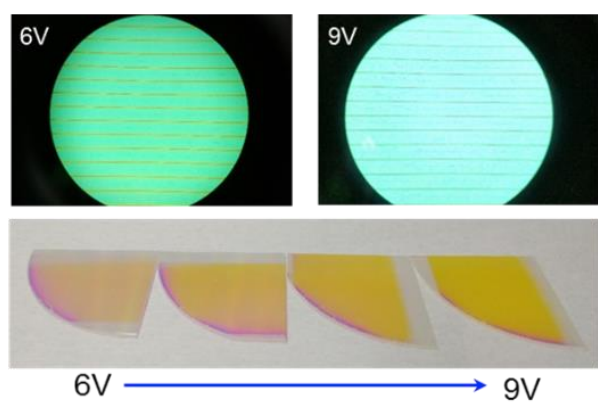

d

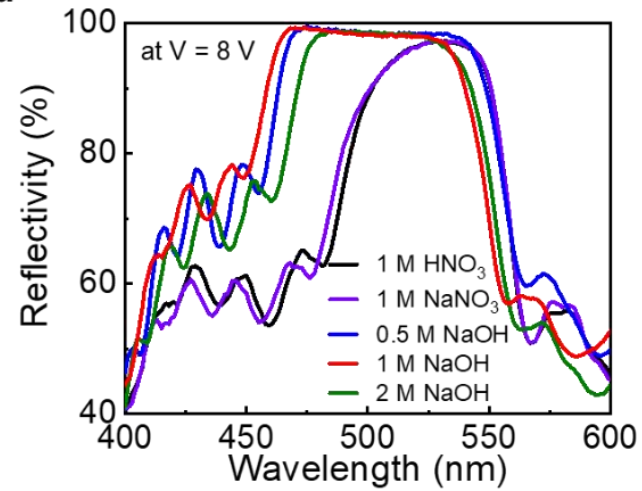

Figure S1. (a) Cross-sectional SEM images of the porous GaN DBR EC etched by $65 \mathrm{wt} \% \mathrm{HNO}_{3}$ at different voltages $(V=6,7,8,9 \mathrm{~V})$. (b) Optical images of the porous GaN DBR EC etched by $65 w \mathrm{t} \% \mathrm{HNO}_{3}$ under different voltages. (c) Cross-sectional SEM images of the porous GaN DBR EC etched by electrolytes with different $\mathrm{pH}$ values and different concentrations. (d) Reflectivity spectra of the porous GaN DBR EC etched at $V=8 \mathrm{~V}$ by electrolytes with different $\mathrm{pH}$ values and concentrations.

To optimize the porous GaN DBR, we first fabricated a large-area porous GaN DBR with a $65 \mathrm{wt} \% \mathrm{HNO}_{3}$ solution at different etching voltages $(V=6,7,8,9 \mathrm{~V})$, which can be precisely controlled by monitoring the supplied voltage during the etching. The corresponding cross- 


\section{WILEY-VCH}

sectional SEM images of the porous GaN layers are shown in Figure S1a. It can be seen that at all voltages, the porous GaN layers are periodic and have uniform size pores. All porous GaN layers maintain a double-layer close-packed structure under $V=8 \mathrm{~V}$ and the pore size increases with increasing the etching voltage. When the etching voltage increases to $V=9 \mathrm{~V}$, the doublelayer porous structure tends to evolve into a single-layer structure by lateral annexation and broadening of nearby pores in $\mathrm{n}^{+}-\mathrm{GaN}$. The porosity of the single-layer porous structure reaches a value of up to $41 \%$, which increases the refractive index difference between the $\mathrm{u}-\mathrm{GaN}$ and $\mathrm{n}^{+}$-GaN layers. In turn, this leads to a high reflectivity of the porous GaN DBR. Figure S1b shows the optical images of the porous GaN DBR obtained at room temperature with $65 \mathrm{wt} \%$ $\mathrm{HNO}_{3}$ solution at different etching voltages.

We studied the effect of the $\mathrm{pH}$ value and concentration of the electrolytes on the morphology of the porous DBR structure. As shown in Figure S1c, at the same etching voltage, the porous GaN DBR etched with $1 \mathrm{M} \mathrm{HNO}_{3}$ or $\mathrm{NaNO}_{3}$ solution has a similar morphology and structure, with a porosity close to $30 \%$. However, the porous GaN DBR etched with a $1 \mathrm{M}$ $\mathrm{NaOH}$ solution exhibits larger triangular porous with a porosity of $40 \%$. The effective refractive index of these $\mathrm{NaOH}$ etched porous $\mathrm{GaN}$ DBRs can be calculated. The results indicate that, at the same etching voltage, the alkali solution $(\mathrm{NaOH})$ is preferable to obtain porous $\mathrm{GaN}$ layers with a larger refractive index difference from bulk GaN, thus facilitating the fabrication of widestopband, high-reflectivity DBRs.

Furthermore, we adjusted the concentration of the $\mathrm{NaOH}$ solution and found that, at the same etching voltage, the concentration of the electrolyte has little effect on the morphology of the porous GaN DBR. However, when the concentration of the alkaline solution reaches $2 \mathrm{M}$, the etching uniformity of DBR decreases and the high concentration alkali solution produces strong secondary etching of the layers.

Figure S1d shows the reflectivity spectra of the porous GaN DBR etched with electrolytes with different $\mathrm{pH}$ values and concentrations. At the same etching voltage and concentration of 


\section{WILEY-VCH}

the electrolytes, the porous $\mathrm{GaN}$ DBR etched with the $\mathrm{NaOH}$ solution has the highest reflectivity (up to 99.5\%) and the widest stopband (about $90 \mathrm{~nm}$ ) centered at $\lambda=500 \mathrm{~nm}$. However, the reflectivity of the porous GaN DBR etched with the $\mathrm{HNO}_{3}$ or $\mathrm{NaNO}_{3}$ solutions coincides in the whole spectrum and shows the same light reflection characteristics with the central wavelength redshifted to $\lambda=532 \mathrm{~nm}$. Furthermore, by increasing the $\mathrm{NaOH}$ solution concentration from 0.5 to $1 \mathrm{M}$, the reflectivity spectrum of the porous GaN DBR blue shifts slightly and the reflectance and stopband do not change much. For a further increase of the $\mathrm{NaOH}$ solution concentration to $2 \mathrm{M}$, the stopband and the reflectivity of the porous GaN DBR deteriorate, which may be due to the instable etching in high concentration alkali solution.

Finally, the effective refractive index of the porous GaN DBR is derived from $n_{\text {eff }}{ }^{2}=[(1$ $\left.\varphi) n_{G a N}{ }^{2}+\varphi n_{a i r}{ }^{2}\right]$, where $\varphi$ is the porosity. This gives a value of $n_{\text {eff }}=1.9$ for the porous GaN described in the main text.

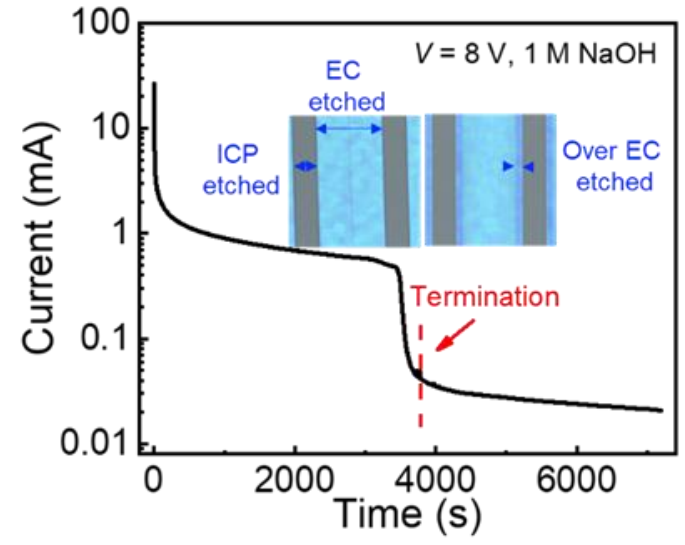

Figure S2. The etching current-time plots at $V=8 \mathrm{~V}$ in $1 \mathrm{M} \mathrm{NaOH}$ solution. Inset: top-view optical microscope images of porous GaN DBRs with different EC etching times (Left: porous GaN DBR wafer etched under precise controlled time of $t=3780 \mathrm{~s}$. Right: porous GaN DBR wafer etched for a longer time $t$ $=5000 \mathrm{~s})$.

In addition, to ensure the etching uniformity of the bottom porous GaN DBR, a Keithley 2400 power supply was used to provide stable etching voltage and the in-situ 


\section{WILEY-VCH}

chronoamperometry technology was used to monitor the etching current over time, as shown in Figure S2. The EC etching of the porous GaN DBR is initially driven by the avalanche effect and enters a steady state when the balance between the oxidation and dissolution of $\mathrm{GaN}$ is reached. ${ }^{[1]}$ As soon as the whole $300 \mu \mathrm{m}$-wide stripe is transformed into the lateral porous $\mathrm{GaN}$, the etching current drops rapidly to a steady state. The etching rate is $\sim 0.04 \mu \mathrm{m} \mathrm{s}^{-1}$ at $V=8 \mathrm{~V}$ in $1 \mathrm{M} \mathrm{NaOH}$ solution. Precise etching time control is also very important to achieve uniform etching and uniform reflection within the porous GaN DBR wafers (insets of Figure S2). If the etching time is insufficient, the $\mathrm{n}^{+}-\mathrm{GaN} / \mathrm{u}-\mathrm{GaN}$ stack cannot be fully transformed into lateral porous GaN DBRs. But if the etching time is too long, the sidewalls of the lateral porous GaN channels are further etched, resulting in non-uniform light reflection and structural instability within the wafer.

\section{S2. Design and optimization of the top dielectric DBR.}

The improvement of the RCEPD performance benefits from the large resonant optical field enhancement as a result of standing waves introduced by the multiple reflections of incident light inside the cavity structure. In this case, the thin active region can serve many times in generating photocarriers, resulting in a high quantum efficiency. The quantum efficiency $\eta$ of a RCEPD is defined as the probability that a single photon incident on the device generates an electron-hole pair which contributes to the detector current as: ${ }^{[2]}$

$$
\eta=\frac{\left(1+R_{2} e^{-\alpha d}\right)}{1-2 \sqrt{R_{1} R_{2}} e^{-\alpha d} \cos \left(2 \beta L+\varphi_{1}+\varphi_{2}\right)+R_{1} R_{2} e^{-2 \alpha d}} \times\left(1-R_{1}\right)\left(1-e^{-\alpha d}\right)
$$

where $R_{1}$ and $R_{2}$ are the reflectivity of top and bottom DBR, respectively, $\alpha$ is the absorption coefficient of the absorption layer, $d$ is the thickness of the absorption layer, $L$ is the cavity length, and $\beta$ is the propagation constant. The quantum efficiency $\eta$ is a strong function of bottom DBR reflectivity $R_{2}$ and near unity reflectivity ( $>99.5 \%$ ) has been realized using our porous GaN DBR in this study. Furthermore, since the propagation constant $\beta\left(\beta=2 n \pi / \lambda_{0}\right.$, 


\section{WILEY-VCH}

where $\lambda_{0}$ is the vacuum wavelength and $n$ is the refractive index) has a wavelength dependence, the quantum efficiency $\eta$ is enhanced periodically at the resonant wavelengths, which occur when $2 \beta=\varphi_{1}+\varphi_{2}=2 m(m=1,2,3 \cdots)$. Thus equation (1) can be rewritten as:

$$
\eta=\frac{\left(1+R_{2} e^{-\alpha d}\right)}{1-2 \sqrt{R_{1} R_{2}} e^{-\alpha d}+R_{1} R_{2} e^{-2 \alpha d}} \times\left(1-R_{1}\right)\left(1-e^{-\alpha d}\right)
$$

When the bottom DBR reflectivity $R_{2}$, the absorption layer thickness and the absorption coefficient are constants for a cavity structure, the quantum efficiency is a function of the top DBR reflectivity $R_{l}$. By only selecting a top DBR with proper reflectivity, the highest optical coupling in the cavity can be achieved to maximize the absorption of the thin active region (only $12 \mathrm{~nm}$ in this study) and compensate the reflection loss of incident light. ${ }^{[3]}$ From the derivative of equation (2) with respect to $R_{l}$, we find that the maximum value of quantum efficiency is $R_{1}=R_{2} e^{-2 \alpha d}$. Since the thickness of the absorption layer is $d=12 \mathrm{~nm}$, the absorption coefficient is $\alpha=1 \times 10^{5} \mathrm{~cm}^{-1}$, and reflectivity of the bottom porous GaN DBR is about $R_{2}=100 \%$ in our experiment, the optimal reflectivity of the top DBR is about $R_{1}=79 \%$. Based on the above calculation, we choose 5 pairs of $\mathrm{SiO}_{2} / \mathrm{Ta}_{2} \mathrm{O}_{5} \mathrm{DBR}$ as the top mirror. The corresponding reflectivity spectrum is shown in Figure 2S. Meanwhile, these two materials have low absorption in visible light and show good mechanical property, chemical stability, as well as excellent adhesion to each other.

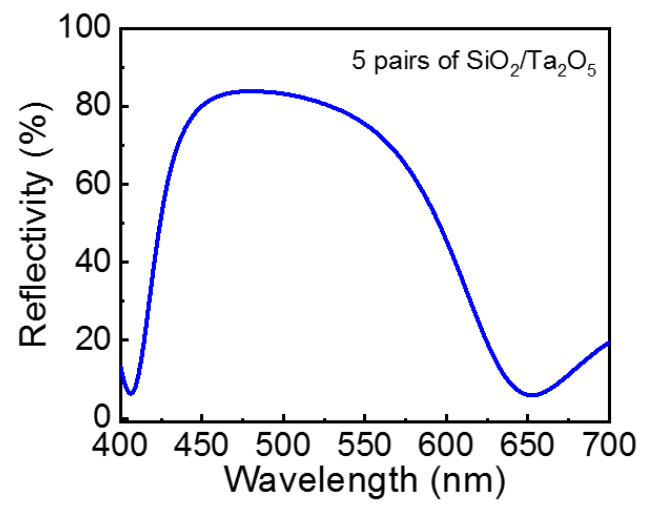

Figure S3. Reflectivity spectrum of the 5 pairs of top $\mathrm{SiO}_{2} / \mathrm{Ta}_{2} \mathrm{O}_{5}$ DBR deposited on glass.

\section{S3. Photoluminescence spectra}




\section{WILEY-VCH}

Figure S4 shows the micro-zone PL spectra of the RCE and the control samples. The control sample shows a broad spectrum due to the inhomogeneous distribution of In in the InGaN/GaN QWs. The emission peak is centered at $\lambda=515 \mathrm{~nm}$ with a FWHM of $43 \mathrm{~nm}$. In contrast, the RCE sample shows three PL peaks at $\lambda=465,500$ and $535 \mathrm{~nm}$. The main PL peak is centered at $500 \mathrm{~nm}$ with a FWHM of $4.8 \mathrm{~nm}$. Furthermore, the PL intensity is 12 times larger than in the control sample.

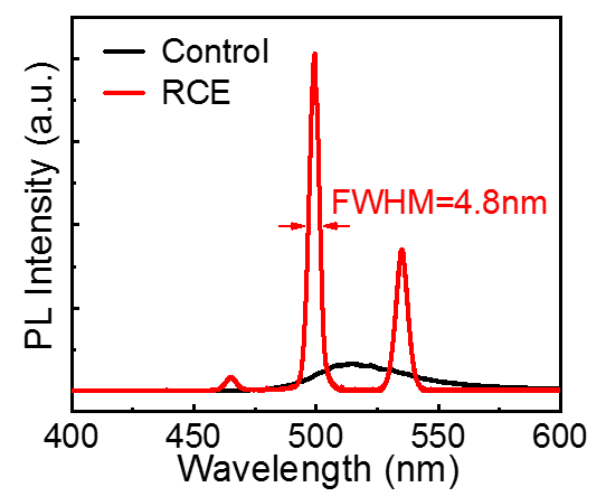

Figure S4. PL spectra of the RCE and control samples at room temperature.

\section{S4. Emission peak distribution within the wafer}
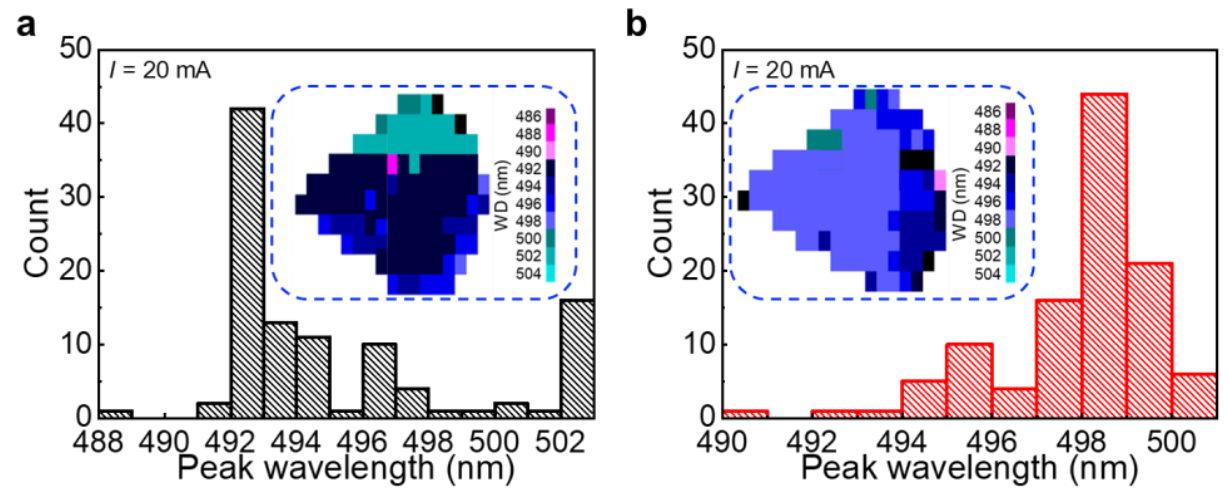

Figure S5. Emission peak wavelength distributions of the control (a) and RCE (b) photodiodes for an injection current of $I=20 \mathrm{~mA}$ within quarter wafers. Insets: the corresponding wavelength distribution (WD) mappings of the control and RCE photodiodes by COW mapping test.

\section{S5. Photocurrent}

The photocurrent $I_{p h}$ has a linear dependence on the light power $P$ over the entire 


\section{WILEY-VCH}

measurement range, as shown in Figure S6. This dependence can be described by the power law, $I_{p h} \propto P^{\alpha}$, where $\alpha=1$ under various applied voltages $(V=0,-1,-2,-3,-4,-5 \mathrm{~V})$, suggesting a linear dependence of the recombination of nonequilibrium carriers on $P .^{[4]}$

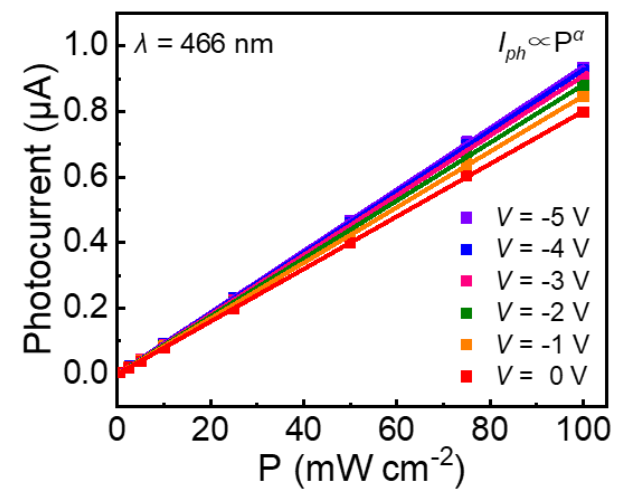

Figure S6. Photocurrents as a function of the light power $P$ at $\lambda=466 \mathrm{~nm}$ under different biases $(V=0,-1$, $-2,-3,-4,-5 \mathrm{~V})$. The solid lines are fits to the measured data.

\section{S6. Energy band diagrams}

a

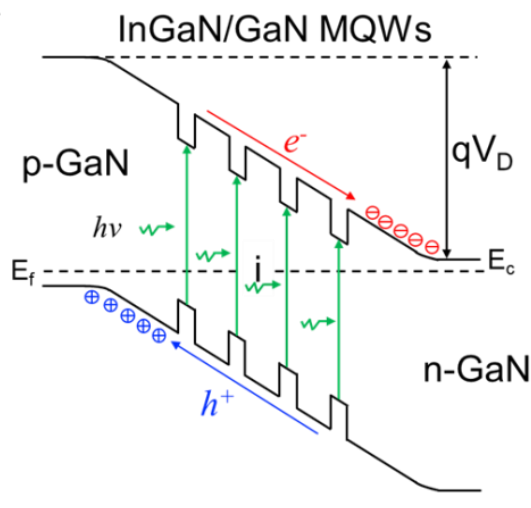

b

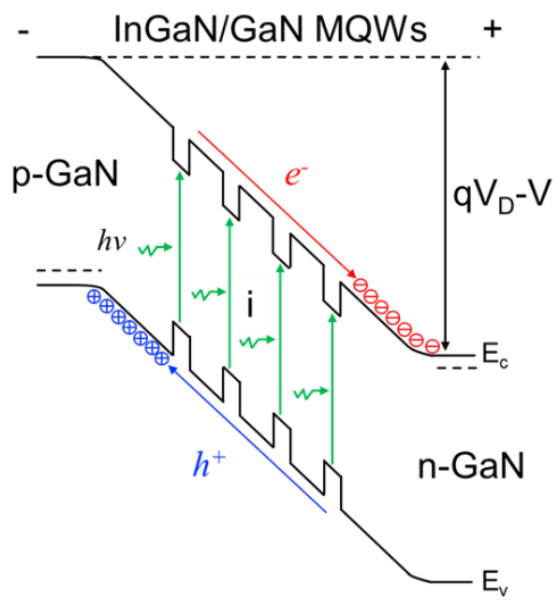

Figure S7. Schematic band diagrams of the PD at (a) zero and (b) reverse bias.

The RCEPD consists of a p-i-n structure. The built-in electric field in the intrinsic (i) region acts to sweep the photo generated carriers in opposite directions under zero bias, as shown in Figure S7a. When the junction is negatively biased, the electric field in the intrinsic region is 


\section{WILEY-VCH}

enhanced, which decreases the carrier transit time, leading to a reduced carrier recombination, as shown in Figure S7b.

\section{S7. Temporal response of the photodiode}

To investigate the frequency characteristics of the RCEPD, we measured the temporal response of the photocurrent at zero bias under different modulation frequencies of visible light from a blue LED (Figure S8a-c). At a frequency of $f=0.5 \mathrm{kHz}$, the rise and decay edges of the time-dependent photoresponse curve are very sharp. The RCEPD can keep in synchronization with the modulation of the optical signal. With increasing $f$ above $2 \mathrm{kHz}$, the optical response of the RCEPD becomes slower, showing rise and fall edges. The corresponding rise and decay times of the RCEPD are 35 and $30 \mu$ s, respectively, longer than those of the control sample (Figure S8d), indicating the EC etching process may introduce additional parasitic resistances in the RCE devices, which will be further addressed in the future. The amplitude of the photocurrent decreases with increasing $f$, as shown in Figure S9a. The decrease of the photocurrent with increasing $f$ becomes less pronounced under an applied reverse bias. As shown in Figure S9b, the response time of the RCEPD tends to decrease with increasing applied voltage. 
a
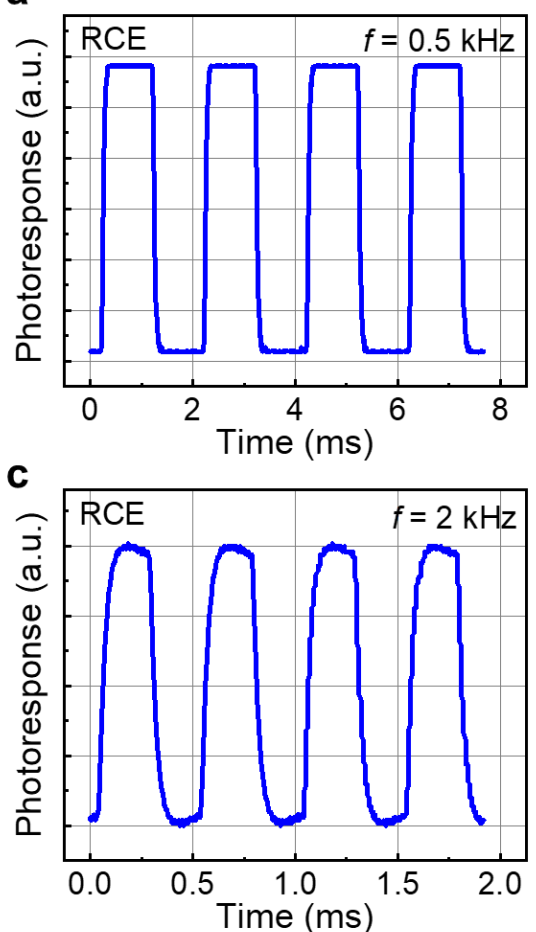

b
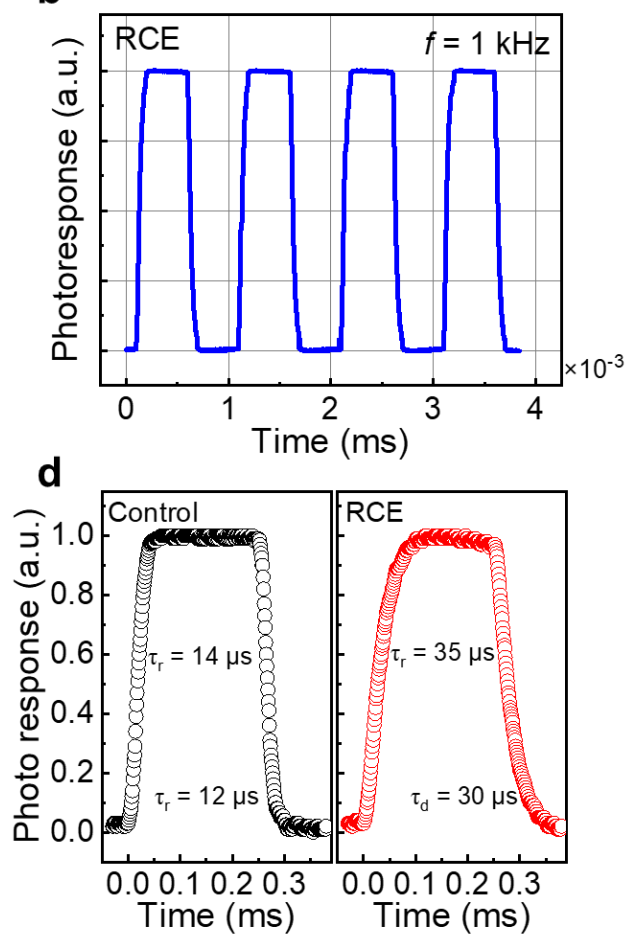

Figure S8. Dynamic response of the RCEPD at different modulation frequencies (a) $0.5 \mathrm{kHz}$, (b) $1 \mathrm{kHz}$, and (c) $2 \mathrm{kHz}$ of light from a blue $\operatorname{LED}\left(\lambda=470 \mathrm{~nm}, P=50 \mathrm{~mW} \mathrm{~cm}{ }^{-2}, V=0 \mathrm{~V}\right)$. (d) Comparison of temporal response of the RCE and control photodiodes at a modulation frequency $f=2 \mathrm{kHz}$.

a

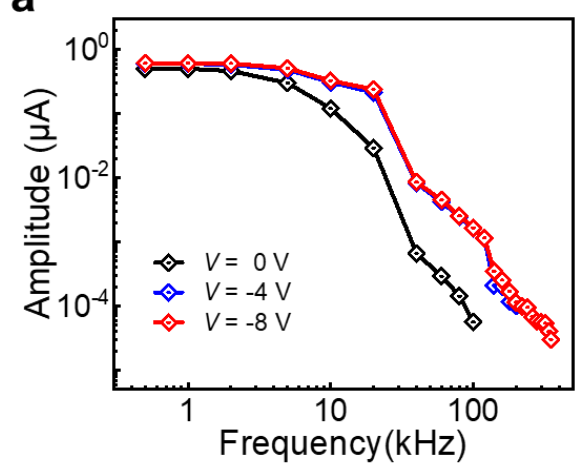

b

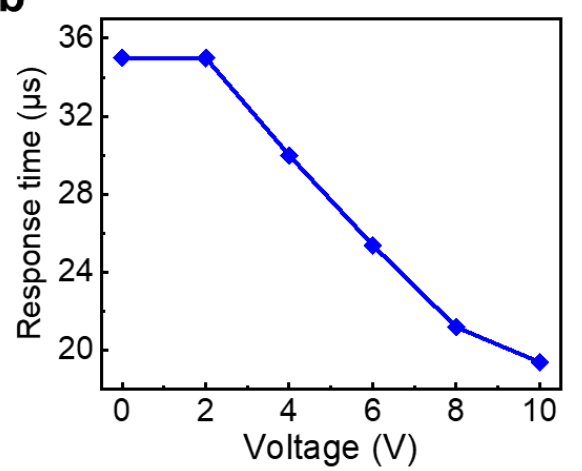

Figure S9. (a) Amplitude of the photocurrent of the RCEPD versus the modulation frequency of visible light

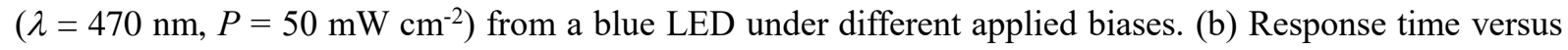
applied voltage of the RCEPD at a modulation frequency of $f=2 \mathrm{kHz}$ from a blue LED ( $\lambda=470 \mathrm{~nm}, P=50$ $\mathrm{mW} \mathrm{cm} \mathrm{cm}^{-2}$. 


\section{WILEY-VCH}
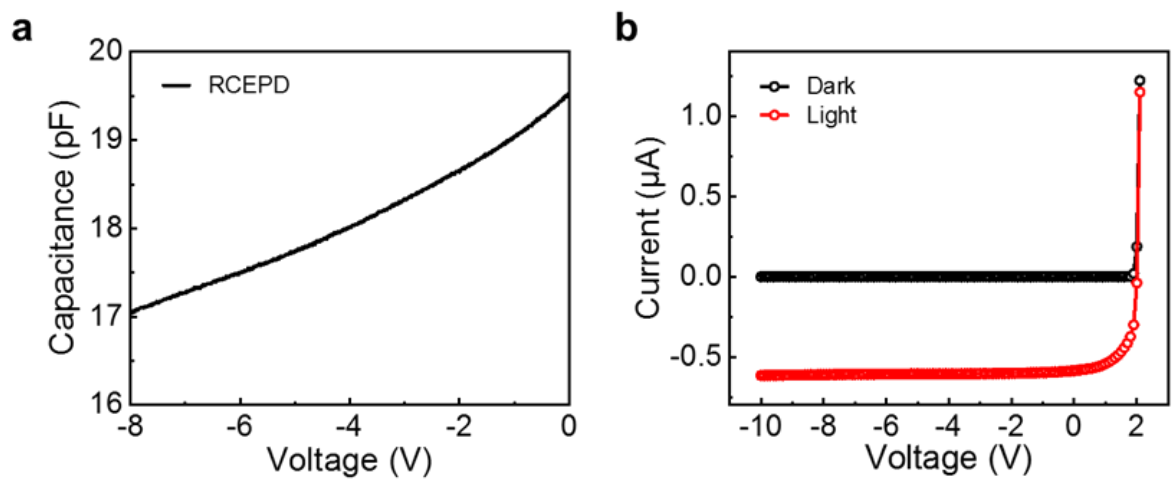

Figure S10. (a) Capacitance-voltage characteristics of the RCEPD. (b) Current-voltage characteristics with and without light of the RCEPD $\left(\lambda=470 \mathrm{~nm}\right.$ and $\left.P=50 \mathrm{~mW} \mathrm{~cm}^{-2}\right)$.

Our experiments demonstrate that the photocurrent of the RECPD can be switched on and off without resorting to any externally applied voltage. We estimate an RC time constant of 1 $\mathrm{ms}$ at $V=0 \mathrm{~V}$, as derived from the measurement of the capacitance $(\mathrm{C}=19 \mathrm{pF})$ and circuit input impedance $(R=53 \mathrm{M} \Omega)$ of the RECPD at $\mathrm{V}=0 \mathrm{~V}$. Both the capacitance and input impedance of the RCEPD have a weak dependence on the applied reverse bias (Figure S10). Based on the above results, the main factor that limits the response speed is the input impedance of the RCEPD. The response time could be improved by decreasing the input impedance, i.e. the parasitic resistances from the porous $\mathrm{GaN}$ DBRs $\left(\mathrm{R}_{\mathrm{DBR}}\right)$ and the $\mathrm{n}-\mathrm{GaN}$ layer $\left(\mathrm{R}_{\mathrm{GaN}}\right)$, by optimizing the porosification of $\mathrm{n}^{+}-\mathrm{GaN}$, increasing the doping of $\mathrm{n}^{+}-\mathrm{GaN}$, optimizing the ohmic contacts of the $\mathrm{p}$ - and n-doped layers, protecting the sidewalls of the mesa, controlling the EC etching voltage and time during the fabrication. In addition, the position and the thicknesses of the MQWs in the cavity could be further optimized to improve the collection of the photogenerated carriers and reduce their transit time across the junction.

\section{References}

[1] C. Yang, L. Liu, S. Zhu, Z. Yu, X. Xi, S. Wu, H. Cao, J. Li, L. Zhao, J. Phys. Chem. C 


\section{WILEY-VCH}

2017, 121, 7331-7336.

[2] M. S. Ünlü, S. Strite, J. Appl. Phys. 1995, 78, 607-639.

[3] Y. F. Lao, G. Ariyawansa, A. G. U. Perera, J. Appl. Phys. 2011, 110 (4), 043112.

[4] A. Roset, Proceedings of the Ire 1955, 43, 1850-1869. 